\title{
The development of codes in public and private language
}

Ss develop systematic, explicit codes to describe repeated occurrences of the same colors and shapes when the description is made for the use of another person. Codes are less explicit and less systematic when descriptions are made for private use in the fairly distant future, and implicit, unsystematic codes are typical of $S s$ making private descriptions for use in the near future. Thus communicative set can influence language; it is suggested that this may have an influence on cognitive activity.

Previous experimental work (Kaplan, 1952; Loewenthal, unpublished) indicates several qualitative and quantitative differences between communicative (public) and self-addressed (private) language. Messages addressed to another person tend to be longer, and to contain terms which are more "communal" and less idiosyncratic than self-addressed messages.

In the present experiment, Ss were asked to make a record of a series of colored shapes as they were exposed one by one. The series was composed of a small set of shapes and colors, with the same shapes and colors recurring frequently in different combinations. It was expected that $\mathrm{Ss}$ might develop some sort of code to represent each color and shape. The purpose of the experiment was to examine differences in the type of code developed between Ss making a record for their own use (private) and Ss making a record for the use of another person (public).

Two "private" groups were used in the experiment. Group $A$ received instructions to record the series so that the record could be used to reconstruct the series in the near future. Group B received instructions to make a record for private use in the fairly distant future.

It was hypothesized that codes developed by Group $B$ would be intermediate in type between those of Group $A$ and the public group (Group C), since the addressee is more "distant."

\section{Subjects}

Ss were 23 undergraduate psychology students (10 men, 13 women). Numbers in each group are shown in Table 1.

\section{Materials}

Seventy $2 \times 2$ in. white cards were employed; in the center of each card was mounted a colored shape. Seven different shapes were used and five different colors (red, yellow, brown, blue, green). There were 35 possible combinations of color and shape; each combination occurred twice in the series. The order of cards was randomized by shuffling.
Ss were provided with a ballpoint pen and colored pencils (red, yellow, brown, blue, green, black) with which to make their records.

\section{Procedure}

Ss were assigned randomly to one of the three groups. Instructions were to record the series of colored shapes so that the record could be used to reconstruct the series exactly as shown. The record was to be for the use of: (Group A) "yourself, immediately after this part of the experiment is over"; (Group B) "yourself, in several weeks' time"; (Group C) "another person."

The cards were then exposed one by one. $\mathrm{S}$ was allowed unlimited time to record the series. Reference back to previously exposed cards was not permitted, but Ss were allowed to refer back to earlier sections of their records.

Results

Inspection of protocols showed that, as expected, Ss appeared to realize that the same shapes and colors were recurring in the series, and different methods of recording were developed. These appeared to be of four types.

I. Explicit code: A "key" is constructed. Each shape is drawn and a letter or number assigned to it. Similarly, a letter or number is assigned to each color. Ss then simply "described" each stimulus by recording the appropriate letters and/or numbers from the code.

II. Explicit code for one attribute only (usually shape): The other attribute is represented by III or IV (see below).

III. Semi-explicit: Ss referred back to earlier, fuller descriptions. A section from a protocol will illustrate this: "blue flower as others"; oval as others but green"

IV. Implicit code: There is no reference to a "key" or to earlier, more detailed descriptions. For example: "Flat sauc; long heart; f.s.; 8 o"

In order of decreasing explicitness, these four types of code may be ranked I - II - III - IV. Each protocol was classified (without knowledge of the experimental group of S) according to which type of code was developed. Table 1 shows the number of $\mathrm{Ss}$ in each group who developed each type of code.

The trend towards increased explicitness over the three groups, $A$ to $B$ to $C$, is significant (Kendall's $S=116, z=3.5, p<.001)$.

Discussion

In public language, Ss developed a systematic, ex- 
Table 1. Numbers of Ss using different types of coding system.

\begin{tabular}{|c|c|c|c|}
\hline $\begin{array}{l}\text { Coding } \\
\text { System }\end{array}$ & $\begin{array}{l}\text { (self-addressed, } \\
\text { near future) }\end{array}$ & $\begin{array}{l}\text { Group } \\
\text { B (self-addressed, } \\
\text { distant future) }\end{array}$ & $C$ (public) \\
\hline I (explicit) & 0 & 1 & 2 \\
\hline \multicolumn{4}{|c|}{ II (explicit only for } \\
\hline one attribute) & 0 & 0 & 4 \\
\hline III (semi-explicit) & 1 & 7 & 1 \\
\hline IV (implicit) & 5 & 1 & 1 \\
\hline$\Sigma$ & 6 & 9 & 8 \\
\hline
\end{tabular}

plicit code for the description of repeated occurrences of the stimuli. This also happened, but to a lesser extent, among Ss making records for their own use in the distant (as opposed to near) future. Ss in Group C tended to develop implicit, or only semi-explicit codes; not only were codes implicit, but they did not exhibit features of systematic organization.

This finding indicates something about the nature of "inner speech." Vygotsky (1934) and others have advanced the view that inner speech plays an important role in the direction of activity, including cognitive activity. In Vygotsky's view, inner speech develops from the egocentric monologues observed in young children. In adults, inner speech is not only selfaddressed, but it is normally silent, and cannot be observed (except by rather inadequate electrophysiological techniques, or by the controversial method of introspection). However, by obtaining samples of selfaddressed descriptions, some indications have been obtained as to the nature of inner speech.

The lack of an explicitly stated code in self-addressed language is presumably associated with the absence of a requirement to explain "what is meant" by a particular abbreviation. However, it can be seen that the development of explicit codes is also associated with a systematic method of recording; communication with another person may therefore lead to a more systematic method of dealing with the material to be communicated. In certain types of problem-solving it might be hypothesized that verbalization (particularly "public" verbalization) would lead to a more systematic approach, thus facilitating problem-solution. (This would apply to only "logical" problems, not to problems of the "creative" variety.) Gagne \& Smith (1962) have in fact demonstrated that verbalization can be associated with more efficient problem-solution, and Loewenthal (unpublished) has shown that this effect is more marked where verbalization is "public" rather than "private."

The relations between language and thinking are very complex, but one approach to the problem is suggested by the present experiment: Language varies with communicative set, and this variation may lead to different ways of "thinking about things."

\section{References}

Gagne, R. M., \& Smith, E. C. The effects of verbalising on problemsolving. J. exp. Psychol., 1962, 63, 12-18.

Kaplan, E. Linguistic characterization of objects in external vs. inner speech. In H. Werner \& B. Kaplan, Symbol formation. New York: Wiley, 1952.

Vygotsky, L. S. Thought and language. English edition (1962) Cambridge, Mass: M.I.T. Press, 1934.

\section{Note}

1. The experiment described in this paper was carried out at University College London, while the author was in receipt of a Medical Research Council Studentship for training in Research Methods. The author is extremely grateful to Dr. P. C. Wason for his helpful advice. 